\title{
Application of matrix product states to the Hubbard model in one spatial dimension
}

\author{
Yukihiro Shimizu $^{1}$, Koji Matsuura ${ }^{1}$ and Hikaru Yahagi ${ }^{1}$ \\ ${ }^{1}$ Department of Applied Physics, Tohoku University, Sendai 980-8579, Japan \\ E-mail:yshmz@snow.apph.tohoku.ac.jp
}

(Received July 12, 2013)

\begin{abstract}
We investigate the application of matrix product states to the Hubbard model in one spatial dimension with both of open and periodic boundary conditions. We develop the variatinal method that the optimization of the variational parameters is carried out locally and sequentially in the framework of matrix product operators (MPO) by including the sign, due to the anti-commutation relation of fermion operators, in the matrix element of MPO. The numerical accuracy of the ground state energy is examined.
\end{abstract}

KEYWORDS: matrix product states, Hubbard model, matrix product operators

\section{Introduction}

The variational method using so-called matrix product states (MPS) as a trial wave function has been developed to understand physics in low dimensional correlated quantum systems $[1,2]$. The method has allowed a deeper understanding of details and problems of the density-matrix renormalization group method, which is believed as one of the most powerful tools for the correlated quantum systems in one spatial dimension. The method of the MPS was originally developed to simulate the quantum spin systems. There are many investigations by using the MPS for the spin systems, however, there are not many applications to the fermionic systems. The negative sign due to the anticommutation relation of the fermion operator has been treated in the framework of the multi-scale entanglement renormalization (MERA) [3,4] or the projected entangled pair states (PEPS) $[5,6]$. In this paper we explain a method that the sign can be exactly treated in the framework of the matrix product operators (MPO). Our method is relatively simple, and it has applicability to the model with long range hopping, suggesting that the treatment of sign in the MPO may be efficient even in the model in two spatial dimension. We will examine the numerical accuracy of the ground state energy.

\section{Matrix Product States and Matrix Product Operators}

\subsection{Open Boundary Condition}

We consider the Hubbard model of $L$ sites in one spatial dimension with the open boundary condition. In order to find a ground state of the model, we employ the variational approach. The trial function, so-called MPS, is assumed as,

$$
|\psi\rangle=\sum_{\alpha_{1}, \alpha_{2}, \cdots, \alpha_{L}} A^{\alpha_{1}} A^{\alpha_{2}} \cdots A^{\alpha_{L}}\left|\alpha_{1}, \alpha_{2}, \cdots, \alpha_{L}\right\rangle,
$$

where $\alpha_{\ell}=0, \uparrow, \downarrow$ or $(\uparrow \downarrow)$ shows the $d$-dimensional local states $(d=4$ for the Hubbard model is called physical dimension) at site, $\ell$. The matrices of $\left\{A^{\alpha_{\ell}}\right\}$ are the variational parameters, that the dimensions of them are $(1 \times d),\left(d \times \min \left(d^{2}, D\right)\right), \cdots,\left(\min \left(d^{L / 2-1}, D\right) \times \min \left(d^{L / 2}, D\right)\right),\left(\min \left(d^{L / 2}, D\right) \times\right.$ 
$\left.\min \left(d^{L / 2-1}, D\right)\right), \cdots,\left(\min \left(d^{2}, D\right) \times d\right),(d \times 1)$, going from $\ell=1$ to $L$. The quantity of $D$ is the bond dimension, which controls the width of variational space.

In order to optimize $A^{\alpha_{\ell}}$ locally and sequentially, we decompose the Hamiltonian into the MPO as,

$$
\begin{aligned}
\mathcal{H}_{\mathrm{OBC}} & =-t \sum_{\ell=1, \sigma}^{L-1}\left(c_{\ell \sigma}^{\dagger} c_{\ell+1 \sigma}+\text { h.c. }\right)+U \sum_{\ell=1}^{L} n_{\ell \uparrow} n_{\ell \downarrow}=\prod_{\ell=1}^{L} W^{[\ell]}, \\
W^{[1]} & =\left(\begin{array}{cccccc}
d_{1} & t c_{1 \uparrow} & t c_{1 \downarrow} & -t c_{1 \uparrow}^{\dagger} & -t c_{1 \downarrow}^{\dagger} & I
\end{array}\right), \\
W^{[2 \leq \ell \leq L-1]} & =\left(\begin{array}{cccccc}
I & 0 & 0 & 0 & 0 & 0 \\
c_{\ell \uparrow}^{\dagger} & 0 & 0 & 0 & 0 & 0 \\
c_{\ell \downarrow}^{\dagger} & 0 & 0 & 0 & 0 & 0 \\
c_{\ell \uparrow} & 0 & 0 & 0 & 0 & 0 \\
c_{\ell \downarrow} & 0 & 0 & 0 & 0 & 0 \\
d_{\ell} & t c_{\ell \uparrow} & t c_{\ell \downarrow} & -t c_{\ell \uparrow}^{\dagger} & -t c_{\ell \downarrow}^{\dagger} & I
\end{array}\right), \quad W^{[L]}=\left(\begin{array}{c}
I \\
c_{L \uparrow}^{\dagger} \\
c_{L \downarrow}^{\dagger} \\
c_{L \uparrow} \\
c_{L \downarrow} \\
d_{L}
\end{array}\right),
\end{aligned}
$$

where $I$ is a unit matrix of $d \times d$, and $d_{\ell}=U n_{\ell \uparrow} n_{\ell \downarrow}$. The matrix elements of $\mathcal{H}_{\mathrm{OBC}}$ are given as,

$$
\begin{aligned}
& \left\langle\alpha_{1}, \cdots, \alpha_{L}\left|\mathcal{H}_{\mathrm{OBC}}\right| \alpha_{1}^{\prime}, \cdots, \alpha_{L}^{\prime}\right\rangle=\sum_{b_{1}, \cdots, b_{L-1}} \prod_{\ell=1}^{L} W_{b_{\ell-1} b_{\ell}}^{\alpha_{\ell} \alpha_{\ell}^{\prime}}, \\
& W_{b_{\ell-1} \alpha_{\ell}}^{\alpha_{\ell} \alpha_{\ell}^{\prime}} \equiv(-1)^{s i g n}\left\langle 0\left|\left(c_{\ell \downarrow}\right)^{n_{\ell \downarrow}\left(\alpha_{\ell}\right)}\left(c_{\ell \uparrow}\right)^{n_{\ell \uparrow}\left(\alpha_{\ell}\right)}\left(W^{[\ell]}\right)_{b_{\ell-1} b_{\ell}}\left(c_{\ell \uparrow}^{\dagger}\right)^{n_{\ell \uparrow}\left(\alpha_{\ell}^{\prime}\right)}\left(c_{\ell \downarrow}^{\dagger}\right)^{n_{\ell \downarrow}\left(\alpha_{\ell}^{\prime}\right)}\right| 0\right\rangle,
\end{aligned}
$$

where $\left(W^{[\ell]}\right)_{b_{\ell-1} b_{\ell}}$ is the $\left(b_{\ell-1}, b_{\ell}\right)$-th matrix element of $W^{[\ell]}$. In order to satisfy the anti-commutation relation of fermion operators, the sign, $(-1)^{\text {sign }}$, should be given as,

$$
(-1)^{s i g n}= \begin{cases}-1 & \text { if } W_{12}^{\alpha_{1} \alpha_{1}^{\prime}}, W_{13}^{\alpha_{1} \alpha_{1}^{\prime}}, W_{14}^{\alpha_{1} \alpha_{1}^{\prime}} \text { and } W_{15}^{\alpha_{1} \alpha_{1}^{\prime}} \text { with } \alpha_{1}^{\prime}=\uparrow \text { or } \downarrow \\ +1 & \text { otherwise }\end{cases}
$$

for $\ell=1$, and

$$
(-1)^{s i g n}= \begin{cases}-1 & \text { if } W_{62}^{\alpha_{\ell} \alpha_{\ell}^{\prime}}, W_{63}^{\alpha_{\ell} \alpha_{\ell}^{\prime}}, W_{64}^{\alpha_{\ell} \alpha_{1}^{\prime}} \text { and } W_{65}^{\alpha_{\ell} \alpha_{\ell}^{\prime}} \text { with } \alpha_{\ell}^{\prime}=\uparrow \text { or } \downarrow \\ +1 & \text { otherwise }\end{cases}
$$

for $2 \leq \ell \leq L-1$, and $(-1)^{\text {sign }}=+1$ for $\ell=L$, respectively.

When we optimize $A^{\alpha_{\ell}}$ sequentially from $\ell=1$ to $L$, and return, and so on, $(\ell=1,2, \cdots, L-$ $1, L, L-1, \cdots, 2,1,2, \cdots)$, the problem that the minimize of $I\left(\left\{A^{\alpha_{\ell}}\right\}\right)=\langle\psi|\mathcal{H}| \psi\rangle /\langle\psi \mid \psi\rangle$ is changed efficiently into a solution of the simple eigen problem of the matrix. We call the path of optimization as a round trip. The optimization process of $A^{\alpha_{\ell}}$ is similar to the optimal algorithm for spin models that is reviewed in section 6 in Ref. [2]. There is no numerical instability at the iterative calculation. The detail of the optimization will be published elsewhere.

\subsection{Periodic Boundary Condition}

We consider two types of the MPS for the periodic boundary condition: One, $\left|\psi^{(\mathrm{I})}\right\rangle$, is identical with the type of MPS for the open boundary condition, as shown in equation (1). Since the matrix product is obtained from the singular value decomposition (SVD) of the eigen vector, if the Hamiltonian could be diagonalized, it is still natural that the form of $\left|\psi^{(\mathrm{I})}\right\rangle$ is assumed as the trial function for the periodic boundary condition. The other, $\left|\psi^{(\mathrm{II})}\right\rangle$, is given as,

$$
\left|\psi^{(\mathrm{II})}\right\rangle=\sum_{\alpha_{1}, \alpha_{2}, \cdots, \alpha_{L}} \operatorname{Tr}\left(A^{\alpha_{1}} A^{\alpha_{2}} \cdots A^{\alpha_{L}}\right)\left|\alpha_{1}, \alpha_{2}, \cdots, \alpha_{L}\right\rangle
$$


where the dimension of all matrices, $A^{\alpha_{\ell}}$, is assumed as $D \times D$. The site independent of dimension of variational parameters may be practical advantage. However, a cost to make the matrix elements, $\left\langle\psi^{(\mathrm{II})}\left|H_{\mathrm{PBC}}\right| \psi^{(\mathrm{II})}\right\rangle$, increases as $d D^{5}$ compared to $d D^{3}$ by assuming type-I MPS, $\left|\psi^{(\mathrm{I})}\right\rangle$.

The MPO for the periodic boundary condition is given as

$$
\begin{aligned}
\mathcal{H}_{\mathrm{PBC}} & =\mathcal{H}_{\mathrm{OBC}}-t \sum_{\sigma}\left(c_{L \sigma}^{\dagger} c_{1 \sigma}+\text { h.c. }\right)=\prod_{\ell=1}^{L} W^{[\ell]}+\prod_{\ell=1}^{L} W^{\prime[\ell]}, \\
W^{\prime[1]} & =\left(\begin{array}{llll}
t c_{1 \uparrow} & t c_{1 \downarrow} & -t c_{1 \uparrow}^{\dagger} & -t c_{1 \downarrow}^{\dagger}
\end{array}\right), \\
W^{\prime[2 \leq \ell \leq L-1]} & =\left(\begin{array}{llll}
I & 0 & 0 & 0 \\
0 & I & 0 & 0 \\
0 & 0 & I & 0 \\
0 & 0 & 0 & I
\end{array}\right), \quad W^{\prime L]}=\left(\begin{array}{c}
c_{L \uparrow}^{\dagger} \\
c_{L \downarrow}^{\dagger} \\
c_{L \uparrow}^{\dagger} \\
c_{L \downarrow}
\end{array}\right),
\end{aligned}
$$

where $W^{\prime[1]}$ and $W^{\prime[L]}$ denote the transfer term between $\ell=1$ and $L$. The matrices, $W^{\prime[2 \leq \ell \leq L-1]}$, are introduced to treat the anti-commutation relation of fermion operators. The matrix elements of $\mathcal{H}_{\mathrm{PBC}}$ are given as,

$$
\begin{aligned}
& \left\langle\alpha_{1}, \cdots, \alpha_{L}\left|\mathcal{H}_{\mathrm{PBC}}\right| \alpha_{1}^{\prime}, \cdots, \alpha_{L}^{\prime}\right\rangle=\sum_{b_{1}, \cdots, b_{L-1}} \prod_{\ell=1}^{L} W_{b_{\ell-1} b_{\ell}}^{\alpha_{\ell} \alpha_{\ell}^{\prime}}+\sum_{b_{1}, \cdots, b_{L-1}} \prod_{\ell=1}^{L} W_{b_{\ell-1} b_{\ell}}^{\prime \alpha_{\ell} \alpha_{\ell}^{\prime}}, \\
& W_{b_{\ell-1} b_{\ell}}^{\prime \alpha_{\ell} \alpha_{\ell}^{\prime}} \equiv(-1)^{s i g n}\left\langle 0\left|\left(c_{\ell \downarrow}\right)^{n_{\ell \downarrow}\left(\alpha_{\ell}\right)}\left(c_{\ell \uparrow}\right)^{n_{\ell \uparrow}\left(\alpha_{\ell}\right)}\left(W^{\prime}[\ell]\right)_{b_{\ell-1} b_{\ell}}\left(c_{\ell \uparrow}^{\dagger}\right)^{n_{\ell \uparrow}\left(\alpha_{\ell}^{\prime}\right)}\left(c_{\ell \downarrow}^{\dagger}\right)^{n_{\ell \downarrow}\left(\alpha_{\ell}^{\prime}\right)}\right| 0\right\rangle .
\end{aligned}
$$

In order to satisfy the anti-commutation relation of fermion operators, the sign, $(-1)^{\text {sign }}$, should be given as,

$$
(-1)^{\text {sign }}= \begin{cases}-1 & \text { if }(1 \leq \ell \leq L-1) \text { and }\left(\alpha_{\ell}^{\prime}=\uparrow \text { or } \downarrow\right) \\ +1 & \text { otherwise }\end{cases}
$$

We note that the treatment of the sign in $W_{b_{\ell-1} b_{\ell}}^{\alpha_{\ell} \alpha_{\ell}^{\prime}}$ is one of the examples to deal with the long range hopping in a Hamiltonian. In the case of the next nearest neighbor or the third nearest neighbor hopping, an extension of $W$, instead of the formula by $W^{\prime}$, may be made easier.

At a naive notion, the optimization of $A^{\alpha_{\ell}}$ in an around path is the most appropriate method for the periodic boundary condition. We will examine the numerical accuracy whether the ground state energy depends on optimization paths, one is the round trip, and the other is the around.

\section{Numerical Results}

As the first test we calculate the ground state energy of non-interacting case, $U=0$, of $L=102$ sites for the open boundary condition. The optimization processes for various bond dimensions are shown in Fig. 1 (a). The energy almost converges after two times round trip optimization. The relative error of the ground state energy in the case of $D=64$ is less than $3 \times 10^{-4}$. In Fig. 1 (b) the optimization process of interacting case, $U=1$, is shown. The convergence behavior is very similar between noninteracting case and interacting one. We may expect similar numerical accuracy in the interacting model. It is consistent that the entanglement (which is measured as the spectrum of singular values of the eigen state for the model that the Hamiltonian of small system can be exactly diagonalized) becomes weak with increasing $U$.

In the case of periodic boundary condition the convergence of the ground state energy varies in the same manner as open boundary condition except lager $D$ is required to reach high accuracy. As 

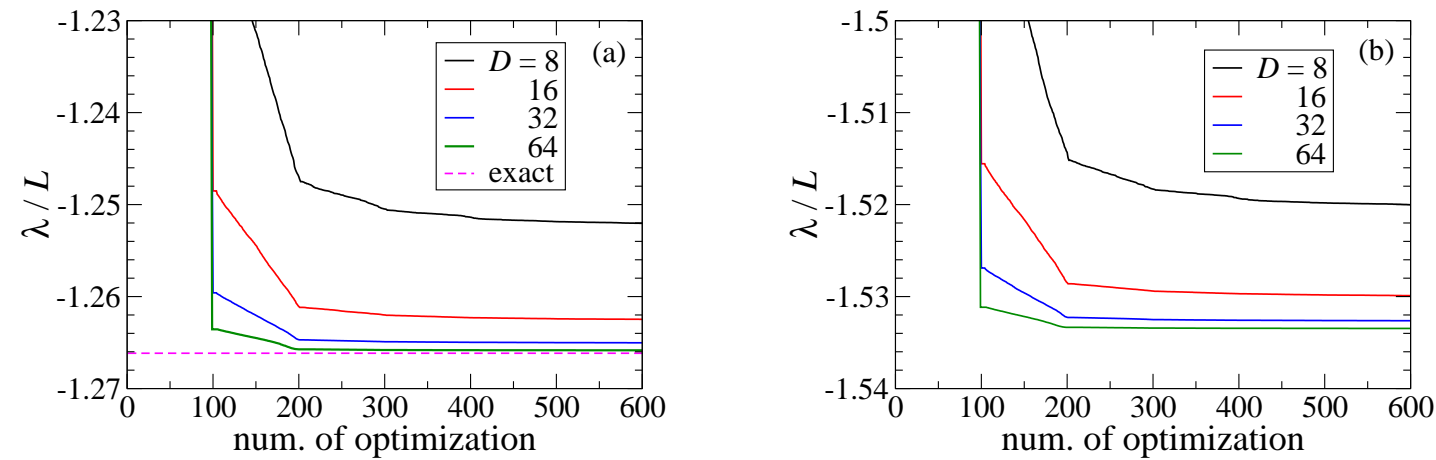

Fig. 1. Optimization process of the ground state energy of the Hubbard model with open boundary condition. The system size is $L=102$. The parameters of the model are $t=1, U=0$ for left figure and $U=1$ for right figure.
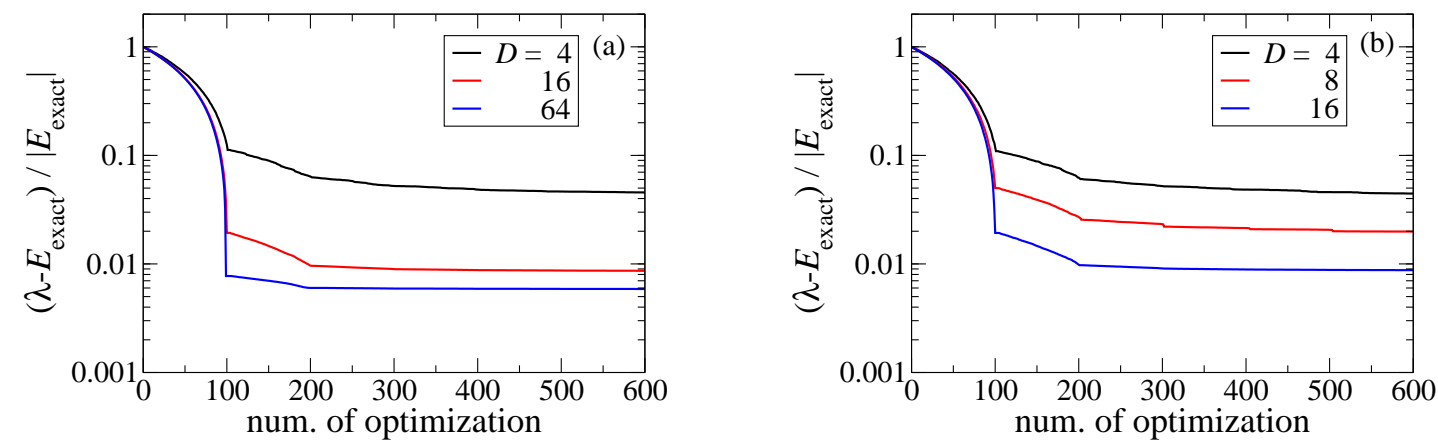

Fig. 2. Relative error of the ground state energy of non-interacting model with periodic boundary condition. The system size is $L=102$. The type-I trial function, $\left|\psi^{(\mathrm{I})}\right\rangle$, is assumed for the left figure. The type-II trial function, $\left|\psi^{(\mathrm{II})}\right\rangle$, is assumed for the right figure.

shown in Fig. 2 (a) the relative error of the ground state energy, which is calculated by employing type-I trial function, $\left|\psi^{(\mathrm{I})}\right\rangle$, and the round trip optimization, is $6 \times 10^{-3}$. If we choose the around optimization, the relative error does not change. When we employ the type-II trial function, $\left|\psi^{(\mathrm{II})}\right\rangle$, as shown in Fig. 2 (b), the numerical accuracy of the ground state energy gets little improvement. The numerical accuracy of the ground state properties will be discussed in another article. We note that there is no numerical instability in the round trip optimization by using type-I trial function. In the case of type-II trial function, there is a little instability, but it does not much of a problem as shown in Fig. 2 (b), due to the solution of the generalized eigen problem.

\section{Conclusions}

We developed the variational method by using the MPS for the Hubbard model with both of open and periodic boundary conditions. The negative sign due to the anti-commutation relation of the fermion operators can be treated in the framework of MPO. Therefore, the variational parameters at each site can be optimized locally. In the case of open boundary condition the numerical accuracy becomes well with increasing the bond dimension of the matrix, $A^{\alpha_{\ell}}$. If the fourfold bond dimension could be used, the relative error for the non-interacting model is reduced to one-tenth. In the case of periodic boundary condition the numerical accuracy does not depend on two types of trial function and optimization paths. In terms of the numerical cost the best way is that the round trip optimization with assuming trial function of $\left|\psi^{(\mathrm{I})}\right\rangle$. The ground state properties, such as the momentum distribution 
and the spin-spin correlation function, will be published in another article.

\section{References}

[1] F. Verstraete, V. Murg and J. I. Cirac: Adv. Phys. 57 (2008) 143.

[2] U. Schllwöck: Ann. Phys. 326 (2011) 96.

[3] P. Corboz and G. Vidal: Phys. Rev. B 88 (2009) 165129.

[4] P. Corboz, G. Evenbly, F. Verstrate and G. Vidal: Phys. Rev. A 81 (2010) 010303(R).

[5] P. Corboz, R. Orús, B. Bauer and G. Vidal: Phys. Rev. B 81 (2010) 165104.

[6] I. Pižon and F. Verstraete: Phys. Rev. B 81 (2010) 245110. 